\title{
Core promoters: active contributors to combinatorial gene regulation
}

\author{
Stephen T. Smale \\ Howard Hughes Medical Institute, Department of Microbiology, Immunology, and Molecular Genetics, and Molecular \\ Biology Institute, University of California, Los Angeles, California 90095-1662, USA
}

In metazoans, thousands of protein-coding genes must be differentially expressed in specific cell types, during development, and in response to a wide variety of extracellular signals. Combinatorial gene regulation strategies are required to generate these diverse expression patterns because only a limited number of transcription factors can be encoded by a limited genome. For a gene to be activated, transcription factors must bind distant control regions and promote the decondensation of repressed chromatin. Then, factors bound to distant control regions and the promoter must stimulate the remodeling of individual nucleosomes and transcription initiation by RNA polymerase II, via effective communication with nucleosome remodeling complexes, coactivator complexes, and the general transcription machinery (Lemon and Tjian 2000). Another important feature of combinatorial regulation is the requirement for several distinct transcription factors to activate a gene (Merika and Thanos 2001). By employing combinations of factors, the number of gene expression patterns that can be achieved is greatly enhanced.

Although combinatorial regulation has been widely studied, one potential contributor has received relatively little attention: the core promoter, which is located between approximately -35 and +35 relative to the transcription start of a metazoan gene. One reason the core promoter generally was not considered to be an active contributor to combinatorial regulation is historical; when the first protein-coding genes were isolated, virtually every gene, regardless of its expression pattern, contained an A/T-rich sequence 25-30 base pairs (bp) upstream of the transcription start site (Breathnach and Chambon 1981). This sequence, with the consensus TATAAA, was called the TATA box. Following the development of functional assays, mutations in TATA boxes were found to reduce transcription initiation and prevent the proper positioning of transcription start sites. Based on these early observations, it was expected that a similar core promoter structure would be found in every cellular gene. The regulation of transcription was expected to rely exclusively on DNA-binding proteins that interact with distal promoters and enhancers.

E-MAIL steves@hhmi.ucla.edu; FAX (310) 206-8623.

Article and publication are at http://www.genesdev.org/cgi/doi/10.1101/ gad.937701.
Today, this simplistic picture of the structure of core promoters for protein-coding genes has been replaced by a level of complexity that is not yet fully understood or appreciated. Most likely, the initial similarity resulted from the fact that the first promoters for RNA polymerase II were identified in DNA viruses and highly expressed cellular genes, which often contain TATA boxes. As more and more promoters for cellular genes have been isolated, the extensive similarity has vanished.

In Drosophila, most core promoters for protein-coding genes fall into two distinct classes (Burke et al. 1998; Kutach and Kadonaga 2000). Roughly half contain a TATA box 25 to $30 \mathrm{bp}$ upstream of the transcription start site combined with an initiator (Inr) element overlapping the start site. The other half contain an Inr element combined with a downstream promoter element (DPE), which is located approximately $30 \mathrm{bp}$ downstream of the start site. Importantly, all three of these elements serve as recognition sites for subunits of transcription factor IID (TFIID), which contains the TATA-binding protein (TBP) and several TBP-associated factors (TAFs) (for review, see Burke et al. 1998; Smale et al. 1998). In mammals, core promoter structure appears to be even more diverse. Precise calculations have been difficult because transcription start sites have been determined accurately for only a small fraction of genes. Nevertheless, the available data suggest that (1) a smaller percentage of mammalian promoters than Drosophila promoters contain TATA boxes, (2) TATA boxes are paired with Inr elements in a smaller percentage of mammalian promoters, (3) DPE elements exist in mammalian promoters, but have been difficult to identify, and (4) many promoters, including a number of promoters within $\mathrm{CpG}$ islands, appear to lack all three of these core elements.

The diversity of core promoter structure leads to two general questions that are of fundamental importance for an understanding of transcriptional control. First, what are the similarities and differences between the mechanisms of transcription initiation catalyzed by the various core promoter classes? Second, why does core promoter diversity exist? Although the first question has been explored in a number of studies (for reviews, see Burke et al. 1998; Smale et al. 1998; Lemon and Tjian 2000), little is known about the second.

One possible reason for core promoter diversity is that the different classes of core promoters may have evolved 
as functionally equivalent landing pads for the general transcription machinery. That is, during the evolution of a complex genome, an interaction site for at least one component of the general machinery may have been required in each promoter, but the recognition site(s) that evolved in a given promoter may have had no special significance other than to facilitate the binding and proper positioning of the general machinery. Early support for this hypothesis was provided by the observation that core promoter elements are functionally interchangeable in the context of simple, synthetic promoters analyzed in cell-free transcription and transient transfection assays. For example, either a TATA box or an Inr element was sufficient for strong, accurately initiated transcription stimulated by transcription factor $\mathrm{Sp} 1$ (O'Shea-Greenfield and Smale 1992). Although TATAdirected transcription initiated $25 \mathrm{bp}$ downstream of the TATA box and Inr-directed transcription initiated from within the Inr itself, the elements were otherwise functionally equivalent.

A second hypothesis to explain the existence of diverse core promoters is that they may be recognized by different proteins or protein complexes, each of which contributes to the transcription of a relatively small percentage of genes. Support for this hypothesis emerged from the properties of TBP-related factors (TRFs; Dantonel et al. 1999; Lemon and Tjian 2000) and TFTC (Wieczorek et al. 1998). The TFTC (ㅍP-free TAFII-containing) complex contains several TAFs in common with TFIID and can substitute for TFIID in cell-free transcription assays, yet TFTC lacks TBP. Although the absence of TBP suggests that TFTC may prefer TATA-less promoters, TFTC and TFIID appear to stimulate transcription with equal efficiency from TATA-containing and TATA-less promoters (Wieczorek et al. 1998). These results suggest that TFTC may not discriminate between core promoters with different structures, although it is likely to make other important contributions to differential gene regulation.

TRF1 recognizes a sequence that differs from the consensus TATA box, perhaps explaining the evolution of a class of promoters containing a non-consensus TATA sequence (Holmes and Tjian 2000). However, most of the diversity within metazoan core promoters appears to involve the variable occurrence of consensus or near-consensus TATA, Inr, and DPE elements. As mentioned above, all three of these consensus elements are recognized by subunits of the ubiquitous TFIID complex. In fact, TFIID complexes purified from cells expressing an epitope-tagged TBP are equally competent for transcription from and/or binding to TATA, TATA-Inr, Inr, and Inr-DPE promoters (Smale et al. 1990; Burke and Kadonaga 1996; Emami et al. 1997). Although these results do not rule out the possibility that the purified preparations contain multiple TBP-containing complexes with different combinations of TAFs or the possibility that alternative complexes are used in a physiological setting, they suggest that the most common core promoter elements serve as recognition sites for the same TFIID complex. Thus, although TRFs contribute to core promoter diversity (Fig. 1D), they are unlikely to be responsible for the variable occurrence of the most common core promoter elements.

The most intriguing hypothesis to explain the variable occurrence of TATA, Inr, and DPE elements is that the diversity makes an important contribution to combinatorial gene regulation. Selective communication between transcription factors bound to distal sites and the core promoter could be of considerable benefit to combinatorial strategies. The results of Butler and Kadonaga (2001) in this issue provide strong support for this hy-
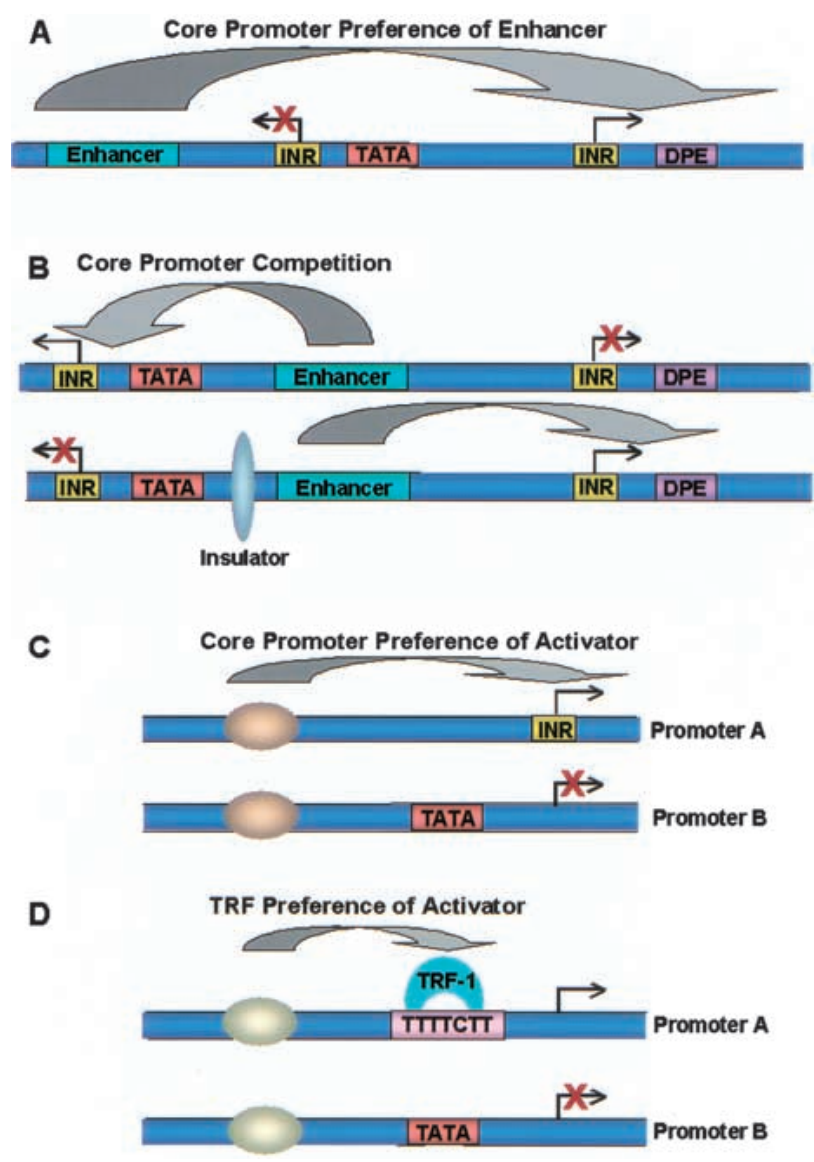

Figure 1. Four strategies by which core promoters may contribute to combinatorial regulation. $(A)$ By possessing intrinsic preferences for specific core promoter classes, some enhancers may influence transcription initiation from only a subset of genes within their stimulatory range (Butler and Kadonaga 2001). (B) As a variation of strategy $A$, competition between core promoters may restrict the stimulatory effects of some enhancers (Ohtsuki et al. 1998). When stimulation of the preferred TATA-Inr promoter is eliminated by insertion of an insulator (Ohtsuki et al. 1998) or, hypothetically, by specific repression of that promoter, the enhancer gains the capacity to stimulate transcription from the Inr-DPE promoter. $(C)$ By possessing intrinsic preferences for a specific core promoter class, some transcriptional activators may stimulate transcription only when bound to promoters that are representative of that class (Ernst et al. 1996; Garraway et al. 1996). (D) As a variation of strategy $C$, some transcriptional activators, and possibly some enhancers, may possess intrinsic preferences for core promoters that interact with TRFs (Holmes and Tjian 2000). 
pothesis. Although a role for core promoter diversity in combinatorial regulation was suggested in previous studies, the current study supports an intriguing new version of the hypothesis and involved an elegant strategy that strengthens the physiological relevance of the results and sets the stage for future advances.

\section{Role of core promoters in restricting the stimulatory capacity of enhancers}

The study by Butler and Kadonaga (2001) was designed to examine one possible strategy by which core promoters in Drosophila could contribute to combinatorial regulation. The hypothesis is based on the knowledge that enhancers can stimulate transcription from promoters at great distances. In a complex genome, multiple enhancers may be in "striking distance" of a given promoter and multiple promoters may be within the stimulatory range of a given enhancer. To optimize the potential for com binatorial regulation, a means of limiting enhancer stimulation to a subset of promoters in its vicinity would be of considerable benefit. Selective communication between promoters and enhancers has been observed previously (Li and Noll 1994; Merli et al. 1996), but the mechanisms responsible for selectivity were not established.

The hypothesis tested by Butler and Kadonaga (2001) was that core promoter diversity contributes to the selective communication between promoters and enhancers. To test this hypothesis, the objective was to determine whether the stimulatory capacity of an enhancer can indeed be restricted to a specific core promoter class. Because the study was performed in Drosophila, the authors were particularly interested in the differences between the TATA-Inr and Inr-DPE classes (Kutach and Kadonaga 2000). Although the properties of a few known enhancers could have been examined in a basic transfection assay, the authors instead designed a strategy that circumvented important obstacles and greatly enhanced the relevance of the results.

One potential obstacle was that only a small subset of enhancers may exhibit core promoter selectivity. To overcome this obstacle, the authors used an enhancer trap strategy and P-element-mediated transformation to introduce promoter-reporter cassettes into numerous locations within the Drosophila genome. The ability to screen a large series of transgenic Drosophila lines greatly increased the probability of identifying enhancer activities that are selective for a TATA-Inr or Inr-DPE core promoter. The second obstacle was that the results would be interpretable only if the two core promoters were examined in precisely the same genomic location. A waffle vector designed by Siegal and Hartl (1997) was ideal for this purpose. The construct that was prepared contained both TATA-Inr and Inr-DPE core promoters, with each core promoter linked to a green fluorescent protein (GFP) reporter gene. When each transgenic line was crossed with another line expressing the Cre recombinase, recombination at loxP sites within the construct resulted in the excision of the Inr-DPE cassette, leaving the TATA-Inr cassette in the genome. When the same lines were crossed with a line expressing the FLP recom- binase, recombination at FRT sites within the construct excised the TATA-Inr cassette, leaving the Inr-DPE cassette in exactly the same genomic location.

From an analysis of 18 pairs of lines, 3 lines expressed much higher concentrations of GFP mRNA from the InrDPE promoter and 1 line expressed much higher concentrations from the TATA-Inr promoter. Equivalent expression from the two promoters was observed in the other lines. An examination of the transcription start sites validated the results. The only reasonable explanation for the different activities was that surrounding control regions, presumably enhancers, preferentially stimulate transcription from one core promoter class. Thus, these results provide strong support for the hypothesis that the two types of core promoters exist, at least in part, for the purpose of limiting enhancer effects to a specific subset of promoters (Fig. 1A).

It may be worthwhile noting one additional implication of the results: They support the notion that distant control regions do not exist solely for the purpose of altering chromatin structure. Rather, the core promoter selectivity can be interpreted as strong evidence that enhancer-bound factors must communicate directly or indirectly with factors bound to the core promoter.

\section{Additional contributions to combinatorial regulation}

Although the results of Butler and Kadonaga (2001) represent compelling evidence that core promoter diversity contributes to combinatorial regulation, initial support for this general hypothesis was provided by previous studies. One notable study by Ohtsuki et al. (1998) tested a hypothesis that was similar to that tested by Butler and Kadonaga. The Ohtsuki study was inspired by the observation that the AE1 enhancer of the Drosophila Hox gene cluster is positioned between the fushi tarazu (ftz) and Sex combs reduced (Scr) promoters, but activates transcription only from the ftz promoter. The ftz promoter contains a consensus TATA box and the Scr promoter, a classic Inr-DPE combination. This observation led to the hypothesis that AE1 preferentially stimulates TATA-containing promoters. Analysis of an extensive series of transgenic Drosophila lines confirmed this hypothesis. Interestingly, however, the TATA preference of the AE1 region was dependent on competition between the two classes of promoters. That is, AE1 was perfectly competent for activation of an Inr-DPE promoter when the nearby TATA promoter was compromised (Fig. 1B).

In the study by Butler and Kadonaga (2001), the enhancer activities that were monitored appear to possess intrinsic core promoter preferences that do not require competition. The authors will need to address the possibility that selectivity requires competition with endogenous promoters in the vicinity of the integration site. A requirement for competition seems unlikely, however, because enhancers with preferences for each core promoter class were detected. In contrast, the enhancers that required competition for selectivity consistently preferred a TATA box.

The promoter for the murine terminal transferase 
(TdT) gene was the focus of another study that suggested a different route through which core promoter diversity can contribute to combinatorial regulation. The -30 region of this promoter is G/C-rich and does not contribute to promoter function (Smale and Baltimore 1989; Garraway et al. 1996). Instead, a consensus Inr element is responsible for core promoter activity and for dictating the location of the transcription start site. Because the in vitro activity of this promoter requires the TFIID complex (Martinez et al. 1995), a reasonable expectation was that a TATA box engineered at -30 would be able to substitute for the Inr at the start site. Surprisingly, when a TATA box was inserted at -30 and the Inr was disrupted by a point mutation, the promoter was completely inactive (Garraway et al. 1996). As a control, Sp1 sites were inserted into the promoter in place of the distal TdT promoter sequences. When transcription was driven by $\mathrm{Sp} 1$ instead of by the natural activators, promoter activity was stronger in the presence of the TATA box than the Inr. These results revealed that the function of the native TdT promoter requires the presence of an Inr and that this preference is due, at least in part, to an Inr preference of transcription factors bound to the distal promoter. Subsequent studies suggested that Elf-1, a critical Ets-family protein that binds $60 \mathrm{bp}$ upstream of the start site, possesses an intrinsic preference for Inrcontaining promoters (Ernst et al. 1996). A DPE-like element located downstream of the Inr also contributes to the preference (Smale et al. 1998).

Because the Inr preference of the TdT promoter was observed in vitro in the absence of an enhancer, this result suggests other means by which core promoters can contribute to combinatorial regulation. The simplest scenario is that core promoter selectivity limits the ability of a transcription factor like Elf- 1 to activate transcription through promoters containing its recognition sequence (Fig. 1C). The consensus recognition site for Elf- 1 is very similar to that of other members of the Ets family, suggesting that DNA-binding specificity is insufficient to restrict Elf-1's functions to the intended set of target genes. By restricting its transactivation capabilities to promoters containing Inr elements, Elf- 1 would be ineffective when bound to promoters containing only a TATA box. Thus, an Ets consensus site within a promoter containing only a TATA box would contribute to activation only when it interacts with a different Etsfamily protein that is competent for TATA-mediated transcription.

In addition to Elf-1, a strong preference for an Inr element has been observed with the glutamine-rich activation domains of Sp1 (Emami et al. 1995). Although interesting from a mechanistic perspective, the biological relevance of this preference is uncertain because fulllength $\mathrm{Sp} 1$ is a potent activator of promoters containing either a TATA box or Inr element (Emami et al. 1995). A preference for activation of TATA-containing promoters was described for c-Fos and a specific activation domain was found to be responsible for this preference (Metz et al. 1994); deletion of this domain resulted in comparable activation of promoters containing either a TATA box or
Inr element. Transcriptional repression by the p53 protein was also found to depend on the presence of a TATA box within the core promoter (Mack et al. 1993). Synthetic promoters containing TATA boxes were potently repressed by $\mathrm{p} 53$, but comparable promoters containing an Inr element instead of a TATA box were resistent to repression.

Finally, some promoters and isolated activators exhibit strong preferences for combinations of core promoter elements, whereas others are equally potent when only one core element is present. For example, strong activation by full-length $\mathrm{Sp} 1$ occurs with a core promoter containing either a TATA box, an Inr element, or both TATA and Inr elements, whereas GAL4-VP16 activates transcription much more strongly when both elements are present (Emami et al. 1995). A similar preference for core promoters containing both elements was observed with the bovine papillomavirus E2 transactivator (Ham et al. 1994). The biological benefit of combining two core elements for the purpose of strengthening a promoter is apparent in the Drosophila Adh gene, which is transcribed from two different promoters. The preferential utilization of the distal promoter in early stages of development is due, at least in part, to the presence of both TATA and Inr elements (Hansen and Tjian 1995). By contrast, the weaker proximal promoter appears to contain only a TATA box. Although these final examples differ in some respects from the preference of an enhancer, promoter, or activator for a specific core element, they help to illustrate the various means by which core promoter diversity can contribute to combinatorial regulation.

\section{Mechanistic basis of core promoter selectivity}

The biochemical mechanisms responsible for the core promoter preferences of enhancers, promoters, and activators remain largely undefined. Therefore, a discussion of this issue must rely primarily on speculation. As mentioned above, the mechanisms responsible for these preferences are not immediately obvious because the TATA box, Inr, and DPE all appear to be recognized by subunits of the same TFIID complex. The most extensive mechanistic insight is perhaps related to the preference of some activators for two core elements (e.g., TATA-Inr) as opposed to one (TATA or Inr). Biochemical studies have shown that a TFIID-TFIIA complex binds cooperatively to the two elements (Emami et al. 1997). This observation suggests that activators like GAL4-VP16 and E2, which prefer two elements, stimulate transcription via a mechanism that benefits from the enhanced affinity of TFIID-TFIIA for the TATA-Inr combination. These activators may be unable to recruit TFIID-TFIIA to weaker core promoters or may be unable to stabilize TFIIDTFIIA binding after recruitment. In contrast, Sp1 activation derives little benefit from the enhanced affinity of TFIID-TFIIA for the TATA-Inr combination, perhaps because it recruits or stabilizes the TFIID-TFIIA complex more strongly than GAL4-VP16 or E2.

Preferences for a specific core promoter element, such as a TATA box, Inr, or DPE, are more difficult to explain. 
It is well established that different TAFs are responsible for selective interactions with different core promoter elements. For example, hTAFII150 and hTAFII250 contribute to the selective recognition of promoters containing Inr elements, and dTAFII60 contributes to the selective recognition of promoters containing DPE elements (Burke and Kadonaga 1997; Chalkley and Verrijzer 1999). In addition, disruption of genes encoding TAFs led to reduced transcription of specific sets of genes, with core promoters responsible for some of the selective effects (for review, see Green 2000). The relevance of these results to the core promoter preferences of enhancers, promoters, and activators is uncertain, however, primarily because the TAFs that have been studied are all components of the same TFIID complex. Unless different classes of core promoters are recognized by different forms of TFIID that carry out selective interactions with transcriptional activators, the TAF results do not provide a clear explanation for the core promoter preferences.

A more attractive hypothesis is that the core promoter preferences involve other differences between the mechanisms of transcription initiation from different core promoter classes. Of particular interest are factors that exhibit unique functions with specific classes of core promoters but that are not considered to be components of the general transcription machinery. The proteins that are most intriguing are TIC-2, TIC-3, and NC2. TIC-2 and TIC-3 were identified as biochemical activities in HeLa cell extracts that were required for transcription from a core promoter containing only an Inr (Martinez et al. 1998). Interestingly, these factors, which have not been cloned or identified, had no effect on the strength of TATA or TATA-Inr core promoters. Although there is no evidence that TIC-2 and TIC-3 contribute to the core promoter preferences of transcriptional activators, their selective involvement in the activity of one core promoter class makes them attractive candidates for this function. It is noteworthy that biochemical studies identified two other factors that are essential for Inr activity, TAFII150 (CIF150) and TIC-1 (Verrijzer et al. 1995; Kaufmann et al. 1996, 1998; Martinez et al. 1998). These factors are less likely to be responsible for the Inr preference of an activator because, unlike TIC-2/3, they appear to stimulate transcription from all core promoter classes.

NC2 is perhaps the most intriguing example of a factor that may be involved in core promoter selectivity. Unlike the factors described above, it possesses opposing activities with TATA-Inr and Inr-DPE promoters. It was originally described as an inhibitor of TATA-Inr promoters (for review, see Maldonado et al. 1999) but was recently purified from Drosophila embryo extracts as an essential and selective activator of Inr-DPE promoters (Willy et al. 2000). The opposing functions of NC2 raise the possibility that it could contribute to the core promoter preference of a transcriptional activator; if the activator stimulates incorporation of NC2 into the preinitiation complex, it most likely would exhibit a strong preference for an Inr-DPE promoter.

Although factors like NC2 and TIC-2/3 have the po- tential to contribute to core promoter preferences of transcriptional activators, selectivity factors are not necessarily required for these preferences. Instead, activators may influence parameters of the transcription initiation reaction that are important for only one core promoter class. For example, the rate-limiting steps for initiation at a TATA promoter may be different from those at an Inr promoter, as suggested previously (Zenzie-Gregory et al. 1992). If an activator influences a step that is rate limiting at only one core promoter class, it would exhibit a preference for that class, even if it does not directly communicate with selectivity factors like NC2 or TIC-2/3. Although most biochemical parameters that have been studied are similar among various core promoter classes, a few differences have been noted (for review, see Smale et al. 1998). One difference is that the reinitiation of transcription appears to be more efficient with TATA-containing promoters than with TATA-less promoters (Yean and Gralla 1997). An activator that selectively enhances the reinitiation frequency may therefore increase the strength of one core promoter class. An alternative possibility is that the conformation of the TFIID complex may differ when it is bound to different core promoter classes, perhaps exposing different surfaces that make the complex competent for activation by distinct subsets of transcriptional activators (e.g., Knutson et al. 2000). A better understanding of the differences between transcription initiation from different classes of promoter, and of the basic mechanisms of transcriptional activation, will be required for a complete understanding of the mechanistic basis of core promoter preferences.

\section{Summary}

The studies cited above provide strong evidence that core promoter diversity is an important contributor to combinatorial regulation. In the future, it will be important to subject this hypothesis to more stringent tests. With respect to the study by Butler and Kadonaga (2001) in this issue, it will be important to identify the enhancers that exhibit core promoter preferences, as well as the promoters that may be relevant targets of those enhancers. Evidence that the endogenous core promoters possess the anticipated structures would provide considerable support for the hypothesis. In addition to confirming the importance of core promoter preferences for combinatorial regulation, it will be important to explore in greater depth the mechanistic basis of these preferences. In some respects, this goal will be difficult to achieve until current controversies regarding the basic mechanisms of transcriptional activation have been resolved. On the other hand, because the core promoter preferences of transcriptional activators lead to a number of testable predictions, further exploration of the mechanisms underlying these preferences may contribute to the resolution of the controversies.

\section{References}

Breathnach, R. and Chambon, P. 1981. Organization and expression of eukaryotic split genes coding for proteins. Annu. Rev. Biochem. 50: 349-383. 
Burke, T.W. and Kadonaga, J.T. 1996. Drosophila TFIID binds to a conserved downstream basal promoter element that is present in many TATA-box-deficient promoters. Genes \& Dev. 10: 711-724.

1997. The downstream core promoter element, DPE, is conserved from Drosophila to humans and is recognized by TAFII60 of Drosophila. Genes \& Dev. 11: 3020-3031.

Burke, T.W., Willy, P.J., Kutach, A.K., Butler, J.E., and Kadonaga, J.T. 1998. The DPE, a conserved downstream core promoter element that is functionally analogous to the TATA box. Cold Spr. Harb. Symp. Quant. Biol. 63: 75-82.

Butler, J.E.F. and Kadonaga, J.T. 2001. Enhancer-promoter specificity mediated by DPE or TATA core promoter motifs. Genes \& Dev. 15: 2515-2519 (this issue)

Chalkley, G.E. and Verrijzer, C.P. 1999. DNA binding site selection by RNA polymerase II TAFs: A TAF(II)250TAF(II)150 complex recognizes the initiator. EMBO $J$. 18: $4835-4845$.

Dantonel, J.C., Wurtz, J.M., Poch, O., Moras, D., and Tora, L. 1999. The TBP-like factor: An alternative transcription factor in Metazoa? Trends Biochem. Sci. 24: 335-339.

Emami, K.H., Navarre, W.W., and Smale, S.T. 1995. Core promoter specificities of the Sp1 and VP16 transcriptional activation domains. Mol. Cell. Biol. 15: 5906-5916.

Emami, K.H., Jain, A., and Smale, S.T. 1997. Mechanism of synergy between TATA and initiator: Synergistic binding of TFIID following a putative TFIIA-induced isomerization. Genes \& Dev. 11: 3007-3019.

Ernst, P., Hahm, K., Trinh, L., Davis, J.N., Roussel, M.F., Turck, C.W., and Smale, S.T. 1996. A potential role for Elf-1 in terminal transferase gene regulation. Mol. Cell. Biol. 16: 6121-6131.

Garraway, I.P., Semple, K., and Smale, S.T. 1996. Transcription of the lymphocyte-specific terminal deoxynucleotidyltransferase gene requires a specific core promoter structure. Proc. Nat1. Acad. Sci. 93: 4336-4341.

Green, M.R. 2000. TBP-associated factors (TAFIIs): Multiple, selective transcriptional mediators in common complexes. Trends Biochem. Sci. 25: 59-63.

Ham, J., Steger, G., and Yaniv, M. 1994. Cooperativity in vivo between the E2 transactivator and the TATA box binding protein depends on core promoter structure. EMBO $\mathrm{J}$. 13: $147-157$.

Hansen, S.K. and Tjian, R. 1995. TAFs and TFIIA mediate differential utilization of the tandem Adh promoters. Cell 82: $565-575$.

Holmes, M.C. and Tjian, R. 2000. Promoter-selective properties of the TBP-related factor TRF1. Science 288: 867-870.

Kaufmann, J., Verrijzer, C.P., Shao, J., and Smale, S.T. 1996. CIF, an essential cofactor for TFIID-dependent initiator function. Genes \& Dev. 10: 873-886

Kaufmann, J., Ahrens, K., Koop, R., Smale, S.T., and Müller, R. 1998. CIF150, a human cofactor for transcription factor IIDdependent initiator function. Mol. Cell. Biol. 18: 233-239.

Knutson, A., Castaño, E., Oelgeschläger, T., Roeder, R.G., and Westin, G. 2000. Downstream promoter sequences facilitate the formation of a specific transcription factor IID-promoter complex topology required for efficient transcription from the megalin/low density lipoprotein receptor-related protein 2 promoter. J. Biol. Chem. 275: 14190-14197.

Kutach, A.K. and Kadonaga, J.T. 2000. The downstream promoter element DPE appears to be as widely used as the TATA box in Drosophila core promoters. Mol. Cell. Biol. 20: $4754-4764$.

Lemon, B. and Tjian, R. 2000. Orchestrating response: A sym- phony of transcription factors for gene control. Genes \& Dev. 14: 2551-2569.

Li, X. and Noll, M. 1994. Compatibility between enhancers and promoters determines the transcriptional specificity of gooseberry and goosebery neuro in the Drosophila embryo. EMBO J. 13: 400-406.

Mack, D.H., Vartikar, J., Pipas, J.M., and Laimins, L.A. 1993 Specific repression of TATA-mediated but not initiator-mediated transcription by wild-type p53. Nature 363: 281-283.

Maldonado, E., Hampsey, M., and Reinberg, D. 1999. Repression: Targeting the heart of the matter. Cell 99: 455-458.

Martinez, E., Zhou, Q., L'Etoile, N.D., Oelgeschläger, T., Berk, A.J., and Roeder, R.G. 1995. Core promoter-specific function of a mutant transcription factor TFIID defective in TATAbox binding. Proc. Natl. Acad. Sci. 92: 11864-11868.

Martinez, E., Ge, H., Tao, Y., Yuan, C.X., Palhan, V., and Roeder, R.G. 1998. Novel cofactors and TFIIA mediate functional core promoter selectivity by the human TAFII150containing TFIID complex. Mol. Cell. Biol. 18: 6571-6583.

Merika, M. and Thanos, D. 2001. Enhanceosomes. Curr. Opin. Genet. Devel. 11: 205-208.

Merli, C., Bergstrom, D.E., Cygan, J.A., and Blackman, R.K. 1996. Promoter specificity mediates the independent regulation of neighboring genes. Genes \& Dev. 10: 1260-1270.

Metz, R., Bannister, A.J., Sutherland, J.A., Hagemeier, C., O'Rourke, E.C., Cook, A., Bravo, R., and Kouzarides, T. 1994. c-Fos-induced activation of a TATA-box-containing promoter involves direct contact with TATA-box-binding protein. Mol. Cell. Biol. 14: 6021-6029.

Ohtsuki, S., Levine, M., and Cai, H.N. 1998. Different core promoters possess distinct regulatory activities in the Drosophila embryo. Genes \& Dev. 12: 547-556.

O'Shea-Greenfield, A. and Smale, S.T. 1992. Roles of TATA and initiator elements in determining the start site location and direction of RNA polymerase II transcription. J. Biol. Chem. 267: 1391-1402.

Siegal, M.L. and Hartl, D.L. 1997. An experimental test for lineage-specific position effects on alcohol dehydrogenase (Adh) genes in Drosophila. Proc. Nat1. Acad. Sci. 95: 1551315518.

Smale, S.T. and Baltimore, D. 1989. The "initiator" as a transcription control element. Cell 57: 103-113.

Smale, S.T., Schmidt, M.C., Berk, A.J., and Baltimore, D. 1990. Transcriptional activation by Spl as directed through TATA or initiator: Specific requirement for mammalian transcription factor IID. Proc. Natl. Acad. Sci. 87: 4509-4513.

Smale, S.T., Jain, A., Kaufmann, J., Emami, K.H., Lo, K., and Garraway, I.P. 1998. The initiator element: A paradigm for core promoter heterogeneity within metazoan protein-coding genes. Cold Spring Harb. Symp. Quant. Biol. 63: 21-31.

Verrijzer, C.P., Chen, J.L., Yokomori, K., and Tjian, R. 1995. Binding of TAFs to core elements directs promoter selectivity by RNA polymerase II. Cell 81: 1115-1125.

Wieczorek, E., Brand, M., Jacq, X., and Tora, L. 1998. Function of TAF(II)-containing complex without TBP in transcription by RNA polymerase II. Nature 393: 187-191.

Willy, P.J., Kobayashi, R., and Kadonaga, J.T. 2000. A basal transcription factor that activates or represses transcription. Science 290: 982-985.

Yean, D. and Gralla, J. 1997. Transcription reinitiation rate: A special role for the TATA box. Mol. Cell. Biol. 17: 3809-3816.

Zenzie-Gregory, B., O'Shea-Greenfield, A., and Smale, S.T. 1992. Similar mechanisms for transcription initiation mediated through a TATA box or an initiator element. J. Biol. Chem. 267: 2923-2830. 


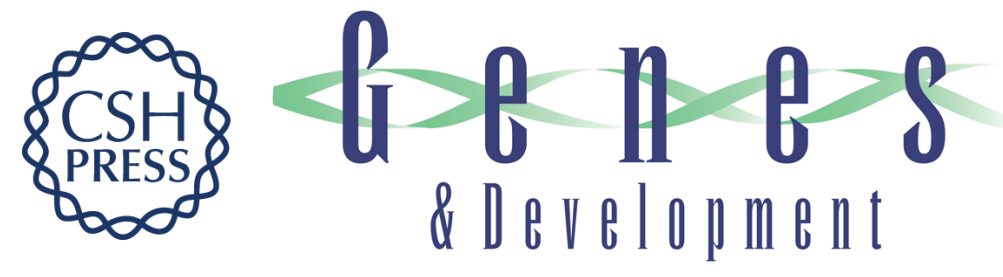

\section{Core promoters: active contributors to combinatorial gene regulation}

Stephen T. Smale

Genes Dev. 2001, 15:

Access the most recent version at doi:10.1101/gad.937701

References This article cites 39 articles, 23 of which can be accessed free at: http://genesdev.cshlp.org/content/15/19/2503.full.html\#ref-list-1

License

Email Alerting Receive free email alerts when new articles cite this article - sign up in the box at the top Service right corner of the article or click here.

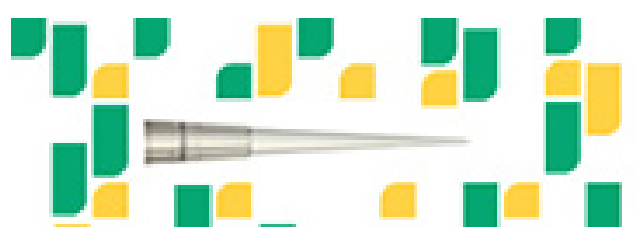

Focused on your science. 